\title{
Chemical Diffusion Coefficient in Dye Sensitized Solar Sells as a Function of Porosity and Surface Roughness
}

\author{
Abdi, Naser ${ }^{1,2}$; Abdi, Yaser,"; ;lemipour, Zahra' ${ }^{1}$; NedaaeeOskoee, Ehsan ${ }^{3}$ \\ 1. Department of Physics, University of Tehran, Tehran 14395, Iran \\ 2. Department of Physics, University of Kurdistan, Sanandaj 6617, Iran \\ 3. Institute for Advanced Studies in Basic Sciences, Zanjan 45137, Iran \\ * Corresponding author. Tel/Fax: +98 2161118610 E-mail address: y.abdi @ut.ac.ir (Y. Abdi)
}

\begin{abstract}
In this work we introduce equations describing the morphological dependency of electron transport in nano-structured solar cells. Using the hopping model for electron transport and using the trap contained diffusion model we define an expression to explain the dependency of the electron diffusion coefficient and conductance versus porosity and surface roughness. Validity of the obtained theoretical formula is examined by a random walk simulation based on hopping model in a fully disordered medium with a random distribution of energy. A Monte-Carlo random walk simulation was used to investigate the effects of morphology on the electron transport in the porous nano-structures. For generation of the random packing of nanoparticles, a stochastic cluster model was utilized. The porosity and surface roughness of the generated porous media were tuned by changing the particle size and the overlap parameters.
\end{abstract}

\section{Introduction}

The porous network of semiconductor nanoparticles such as $\mathrm{TiO}_{2}$ nanoparticles is used as the photo-anode in dye sensitized solar cells. Performance of the solar cells is affected by the electron transport in this porous media ${ }^{1-4}$. Experimental investigations show that the electrical characteristics of the cells depend on the morphological parameters ${ }^{5,6}$. Monte-Carlo random walk (MCRW) simulations was recently used to study electron transport in porous media ${ }^{7-9}$. Cass et al. ${ }^{10,11}$ have studied the effect of the electrical connectivity between the nanoparticles on the transport properties of the electrons. Anta et al. ${ }^{7}$ have utilized MCRW simulations to investigate the effect of nanoparticle size on the electron transport in a random packing network of nanoparticles. They have used an exponential distribution for trap energies. Their results based on the $\mathrm{r}^{2}$ model, in which traps are placed on the surface of the nanoparticles, depict an increase in the diffusion coefficient by increasing the particle size (as obtained by experiments) ${ }^{5,6}$. In this work we have used $\mathrm{r}^{2}$ model for simulations of the transport to illuminate porosity and surface roughness dependency of the electron transport.

Two main approaches have been used to explain electron transport in the trap contained nanostructured media. The first one is the multiple trapping (MT) model. In this model, transport occurs by the electrons in the conduction band and the diffusion of the electrons is affected by multiple trapping events. MT has been a successful model to explain the transport properties in metal-oxide semiconductors used in solar cells ${ }^{12}$. The second approach is the hopping transport model $^{13}$. In the hopping transport model, direct transition between the localized trap states occurs. 
Thermal activation and quantum mechanical tunneling are assumed to be responsible for such transitions. The hopping model is widely used to describe electron transport in the nano-crystalline solar cells. The hopping model appears to well describe the experimental results of the chemical diffusion coefficient obtained from high-efficiency nano-crystalline solar cells.

In this work we have used the hopping model to obtain the electrical parameters of the nanostructured network versus the morphological parameters. In the previous works ${ }^{7-13}$ dependency of the chemical diffusion coefficient on the morphological parameters was reported. The main advantage of this work is obtaining the porosity/surface roughness dependent equations for the chemical diffusion coefficient. Derivation of an analytical relationship between the morphological parameters, and the chemical diffusion coefficient is reported here for the first time.

\section{Theoretical calculations for morphology dependent electron transport}

\subsection{Chemical capacitance}

Considering chemical and electrical potentials $\mu$ and $\varphi$, electrochemical potential can be written as $\eta=\mu-\mathrm{e} \varphi$. By ignoring the variation of the electrical potential, variation of the Fermi level can be identified by $\mathrm{d} E_{f}=\mathrm{d} \mu$. The chemical capacitance (per unit volume) is defined as ${ }^{14,15}$ :

$$
C_{\mu}=q^{2} \frac{d n}{d \mu}
$$

The energies of the trap states which are distributed homogeneously on the surface of the porous media are taken from the usual exponential distribution ${ }^{14}$ :

$$
g_{l}(E)=\frac{n_{t r}}{K_{B} T_{0}} \exp \left[\left(E-E_{c}\right) / K_{B} T_{0}\right]
$$

where $n_{t r}, K_{B} T_{0}$, and $E_{c}$ are the density of traps, characteristic energy of traps, and the conduction band energy level, respectively. In the zero-temperature limit, the chemical capacitance of the localized states can be written as ${ }^{17}$ :

$$
C_{\mu}^{l}=q^{2} g_{l}\left(E_{f}\right)=\frac{q^{2} n_{t r}}{K_{B} T_{0}} \exp \left[\left(E_{f}-E_{c}\right) / K_{B} T_{0}\right]
$$

where $\mathrm{q}$ is the positive elementary charge. Capacitance of the extended states at a temperature of, $\mathrm{T}$, is also expressed as ${ }^{18}$ :

$$
C_{\mu}^{0}=\frac{q^{2} N_{0}}{K_{B} T} \exp \left[\left(E_{f}-E_{c}\right) / K_{B} T\right](4)
$$

where $N_{0}$ is the effective density of the extended states.

\subsection{Diffusion coefficient and electrical conductivity}

The jump diffusion coefficient that can be calculated from the simulation is defined as ${ }^{19,20}$ :

$$
D_{J}=\frac{1}{6 t}\left\langle\left(\frac{1}{N} \sum_{i=1}^{N} \Delta r_{i}\right)^{2}\right\rangle
$$


where $\Delta r_{i}$ is the displacement of the ith particle at time $\mathrm{t}$, and $\langle>$ denotes a statistical average. According to the Darken equation, the chemical diffusion coefficient, $D_{n}$, can be expressed as ${ }^{20}$, 21 :

$$
D_{n}=\chi_{n} D_{J}
$$

where the thermodynamic factor, $X_{n}$, is defined as follows:

$$
\chi_{n}=\frac{n}{K_{B} T} \frac{d \mu}{d n}=\frac{q^{2} n}{K_{B} T} \frac{1}{C_{\mu}}
$$

$C_{\mu}=C_{\mu}^{0}+C_{\mu}^{l}$ is the total capacitance. $\chi_{n}$ for the distribution of Equation 2 has a constant value of $\frac{T_{0}}{T}$. Jump diffusion coefficient for hopping model can be expressed in terms of mean effective jump frequency, $\langle v\rangle$, and the square of the effective jump length, $\left\langle r^{2}\right\rangle$, as ${ }^{22}$

$$
D_{J}=\frac{1}{6}\langle v\rangle\left\langle r^{2}\right\rangle
$$

To achieve the transition rate between the trap states one can use Miller-Abrahams expression for the jump frequency, $v$, which can be written as $^{23}$

$$
v=\left\{\begin{array}{c}
=v_{0} \exp \left[-2 \frac{r}{\alpha}-\frac{E_{j}-E_{i}}{k_{B} T}\right]\left(E_{j}>E_{i}\right) \\
=v_{0} \exp \left[-2 \frac{r}{\alpha}\right]\left(E_{j} \leq E_{i}\right)
\end{array}\right.
$$

where $\alpha$ is the localization radius. Effective jump length, $\langle r\rangle$, in the bulk material with a continuous media, depends on the transport level and characteristic energy of the trap states. The jump length, $r$, is not a fixed length, but is a random variable. In the models with the different spatial distributions of traps, the variation range of the jump length is different. There is a well-known model, in which the discrete-jump problem was studied by assuming the fixed-length jumps of length, $\langle r\rangle$, with random angles (the so-called Rayleigh flights) ${ }^{24}$. According to the Rayleigh flights model, $\langle r\rangle$ is the radius of sphere which includes just one trap. As a result, assuming a constant volume density of $\mathrm{n}_{\mathrm{tr}}=\frac{N_{\text {tr }}}{V}$ for the static traps leads to have the following relationship

$$
\frac{4}{3} \pi\langle r\rangle^{3}=\frac{V}{N_{t r}}
$$

On the other hand, effective jump length in the porous structures must be depended not only on the transport level and characteristic energy of the trap states, but also on the morphology of the transport media. The jump length in the porous media is higher than the continuous ones. As stated before, the different spatial distribution for the traps leads two different ranges of jump length variations. But, the effective jump length does not depend on the trap distribution ( $r^{2}$ or $r^{3}$ models). The following relationship can be written for the effective jump length for the porous materials 


$$
\frac{4}{3} \pi\langle r\rangle^{3}=\frac{V}{N_{t r}}=\frac{V_{\text {occupied }}+V_{\text {voids }}}{N_{t r}}=\frac{1+\frac{V_{\text {voids }}}{V_{\text {occupied }}}}{\frac{N_{\text {tr }}}{V_{\text {occupied }}}}=\frac{1+\frac{V_{\text {voids }}}{V_{\text {occupied }}}}{n_{\text {tr }}}
$$

where $V_{\text {occupied }}$ and $V_{\text {voids }}$ are the volume of occupied and voids in the porous media with the porosity of $P$ and $N_{t r}$ is number of traps in the media with the volume of $V$. Considering $P=\frac{V_{\text {voids }}}{V}$ , equation 11 follows that

$$
\frac{4}{3} \pi\langle r\rangle^{3}=\frac{1+\frac{V_{\text {voids }} / V}{1-V_{\text {voids }} / V}}{n_{t r}}=\frac{1}{(1-P) n_{t r}}
$$

By approaching the porosity, $P$, to zero the effective jump length approaches to its value in continuous media. Substituting $n_{t r}=n_{0} \exp \left(\frac{E_{t r}-E_{c}}{k_{B} T_{0}}\right)$ into Equation 10 we arrive at the following results for the effective jump length

$$
\langle r\rangle=\left[\frac{4}{3} \pi n_{0}(1-p)\right]^{-1 / 3} \exp \left(-\frac{E_{t r}-E_{c}}{3 k_{B} T_{0}}\right)
$$

The energy level, $E_{t r}$, in this equation is a pure electrical parameter that optimizes the upward jump, $\left(E_{j}>E_{i}\right)$.

The transport energy, $E_{t r}$, can be obtained by inserting the morphology independent effective jump length of $\left(\frac{4}{3} \pi n_{0}\right)^{-1 / 3} \exp \left(-\frac{E_{t r}-E_{c}}{3 k_{B} T_{0}}\right)$ into Equation 9 and finding the optimize energy. Inserting the effective jump length and finding the optimize energy, we arrive at the result

$$
E_{c}=E_{t r}+3 k_{B} T_{0} \ln \left[\frac{3 \alpha T_{0}}{2 T}\left(\frac{4 \pi n_{0}}{3}\right)^{1 / 3}\right]
$$

Substituting Equation 14 into Equation 13 gives the following expression for the effective jump length

$$
\langle r\rangle=\frac{3 T_{0}}{2 T} \alpha(1-P)^{-1 / 3}
$$

Using Equation 9 and the distribution function of Equation 2, $\langle v\rangle$ for the states below the transport level can be expressed as ${ }^{22}$

$$
\langle v\rangle=\int_{E_{f}}^{E_{t r}}\left\{v_{0} \exp \left[-2 \frac{\langle r\rangle}{\alpha}-\frac{E_{t r}-E}{k_{B} T}\right]\right\} g_{l}(E) f\left(E-E_{f}\right) d E
$$

where $f\left(E-E_{f}\right)$ is the Fermi-Dirac function. Substituting Equations 15 and 16 and calculating the integral of Equation 16, we arrive at the following result for jump diffusion coefficient.

$D_{j}=\frac{1}{6} v_{0}\left(\frac{3 \alpha T_{0}}{2 T}\right)^{2}(1-P)^{-2 / 3}\left(1-\frac{T}{T_{0}}\right) \exp \left\{-3 \frac{T_{0}}{T}(1-P)^{-1 / 3}-\left(E_{t r}-E_{f}\right)\left[\frac{1}{k_{B} T}-\frac{1}{k_{B} T_{0}}\right]\right\}$

The chemical diffusion coefficient, $D_{n}$, is also can be obtained by substituting the Equation 17 into Equation 6. In experimental studies ${ }^{5,6}$ measurement of chemical diffusion coefficient is carried out at a constant light intensity, which leads to a constant photo-charge. In this situation it is proper 
that we rewrite the chemical diffusion coefficient as a function of photo-electron density of $n_{p h}=$ $n_{t r} \exp \left(\frac{E_{f}}{k_{B} T_{0}}\right)$.

$D_{n}=\frac{1}{6} v_{0}\left(\frac{3 \alpha}{2}\right)^{2}\left(\frac{T_{0}}{T}\right)^{3}(1-P)^{-2 / 3}\left(1-\frac{T}{T_{0}}\right)\left(\frac{n_{p h}}{n_{t r}}\right)^{\left(\frac{T_{0}}{T}-1\right)} \exp \left\{-3 \frac{T_{0}}{T}(1-P)^{-1 / 3}-E_{t r}\left[\frac{1}{k_{B} T}-\frac{1}{k_{B} T_{0}}\right]\right\}$

Ansari et al. show in their work that RW simulation result is consistent with the theoretical formula in which $n_{p h}$ and $n_{t r}$ are the surface densities and not volume densities ${ }^{9}$. According to their achievement, density of traps, $n_{t r}$, and photo-electron density, $n_{p h}$, in Equation 16 can be replaced by the surface density of traps, $n_{t r}^{S}$, and surface photo-electron density, $n_{p h}^{S}$, respectively. The surface roughness $\xi(=\mathrm{S} / \mathrm{V})$ is an important parameter in the porous media. In the $\mathrm{r}^{2}$ model, in which traps are placed on the surface of the nanoparticles, $n_{p h}^{S}$ depends on the surface roughness as: $n_{p h}^{S}=\xi^{-1} n_{p h}$. Consequently, Equation 18 can be rewritten as

$D_{n}=\frac{1}{6} v_{0}\left(\frac{3 \alpha}{2}\right)^{2}\left(\frac{T_{0}}{T}\right)^{3}(1-P)^{-2 / 3}\left(1-\frac{T}{T_{0}}\right)\left(\frac{n_{p h}^{s}}{n_{t r}}\right)^{\left(\frac{T_{0}}{T}-1\right)} \zeta^{\left(1-\frac{T_{0}}{T}\right)} \exp \left\{-3 \frac{T_{0}}{T}(1-P)^{-1 / 3}-E_{t r}\left[\frac{1}{k_{B} T}-\frac{1}{k_{B} T_{0}}\right]\right\}$

The electrical conductivity can be expressed in terms of the chemical diffusion coefficient and chemical capacitance as:

$$
\sigma_{n}=q^{2} D_{n} \frac{d n}{d \mu}=D_{n} C_{\mu}
$$

\section{3- Random walk numerical simulation for hopping transport}

To simulate a mesoporous $\mathrm{TiO}_{2}$ as a transport media we have used the random-cluster model. The cluster model algorithm works as follows: the procedure is started by putting a sphere (nanoparticle) with radius $r$ at the origin. In this simulation nanoparticle size, $r$, is assumed to be $20 \mathrm{~nm}$. It was shown in the experimental reports ${ }^{5,6}$ that the suitable nanoparticle size for maximum efficiency of solar cells is around $20 \mathrm{~nm}$. Then, spheres with the same radius are being brought randomly around the initial sphere to cover the initial one's surface. This process is repeated so that several shells of spheres are added to the initial sphere to form a bigger sphere. We have placed the spheres one by one on the condition that they have to be at a minimum distance $d_{r}$ from at least one other sphere. The value of $d_{r}$ is taken to be smaller than $2 r$, to make an overlap between the nanoparticles. A realistic mesoporous media produced by this algorithm is shown in Figure 1. To avoid the finite size effects the periodic boundary condition was utilized. Periodic boundary condition is often chosen in the numerical simulations ${ }^{16,26,27}$ for approximating a large (infinite) system by using a small part of the system. In this condition, when an electron passes through one side of the cell, it re-appears on the opposite side.

In the next step, static traps were placed on the surface of the obtained porous network with a surface density of $n_{0}^{S}=1 \mathrm{~nm}^{-2}$. The energies of the traps are taken from the exponential distribution of Equation 2. 
In the single electron random walk simulation an electron is allowed to jump to a neighboring trap with the minimum hopping time for the possible jump. The hopping times for jumping the electron from trap $i$ to trap $j$ are calculated from the Miller-Abrahams equation ${ }^{24}$.

$$
T_{i j}=-\ln (p) 1 / v_{0} \exp \left[2 \frac{x}{\alpha}+\frac{E_{i}-E_{j}}{2 K_{B} T}+\frac{\left|E_{i}-E_{j}\right|}{2 K_{B} T}\right]
$$

Where $p$ is a random number distributed uniformly between 0 and $1, v_{0}$ is the attempt-to-jump frequency, $\mathrm{x}$ is the distance between the traps, $\alpha$ is the localization radius, and $E_{j}\left(E_{i}\right)$ are the energies of the target (starting) traps. In each simulation step, an electron is allowed to jump to a neighboring trap with the minimum hopping time for the possible jump, and the corresponding hopping times of $T_{i j}$ is added to the total time of simulation. For a normal diffusion, after a well enough long time, the diffusion coefficient can be calculated by:

$$
D_{j}=\frac{\left\langle\left(X_{t}-X_{0}\right)^{2}\right\rangle}{6 t}
$$

$D_{j}$ is the jump diffusion coefficient and the symbol $<>$ denotes a statistical average.

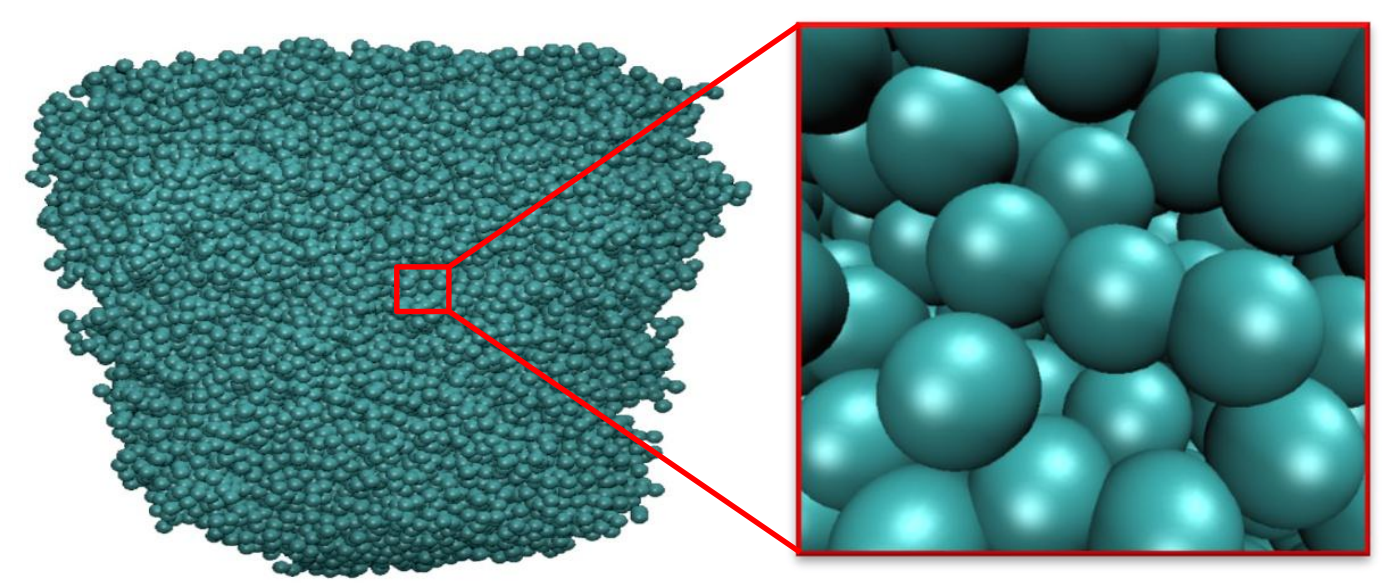

Figure 1.Visualization of the network produced by the random cluster model.

\section{Result and discussion}

To investigate the validity of the equation 17 , the random walk simulation is carried out for networks with different porosities and different surface-roughness values at a same Fermi-level, $E_{f}=-0.7 \mathrm{eV}$. The values of constants used for the simulations are given in Table 1. Results are presented in Figure 2. Figure 2.a and 2.b show chemical diffusion coefficient and conductivity of the porous media as a function of porosity. Solid lines are obtained by Equation 17 and points are the simulation results. Figure 2.c and 2.d represent the diffusion coefficient and conductance versus the surface roughness, $\zeta$. As shown in these figures at a constant fermi level, electron transport is independent of surface roughness. The same results can be obtained by MT model which have been carried out previously ${ }^{9,28}$. It was shown by Ansari et al that $D_{n}$ is independent of $\zeta$ at the constant Fermi level. It must be noted that the experimental values for the diffusion coefficient which are usually obtain by photocurrent transient measurement are resulted from measurements at constant photo-charge density. 


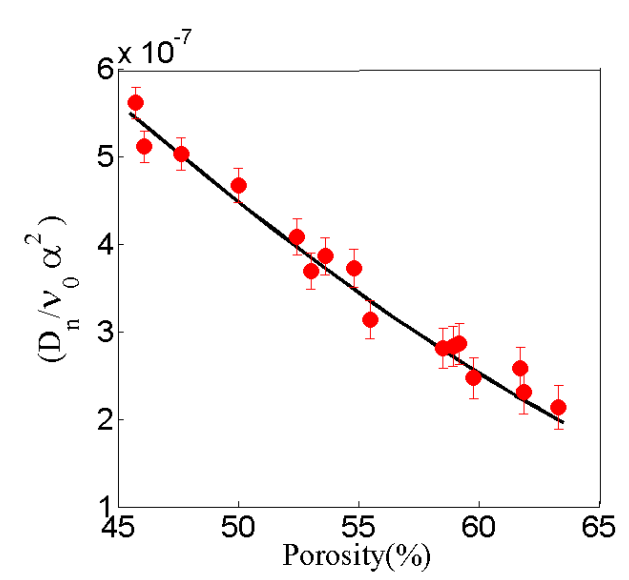

(a)

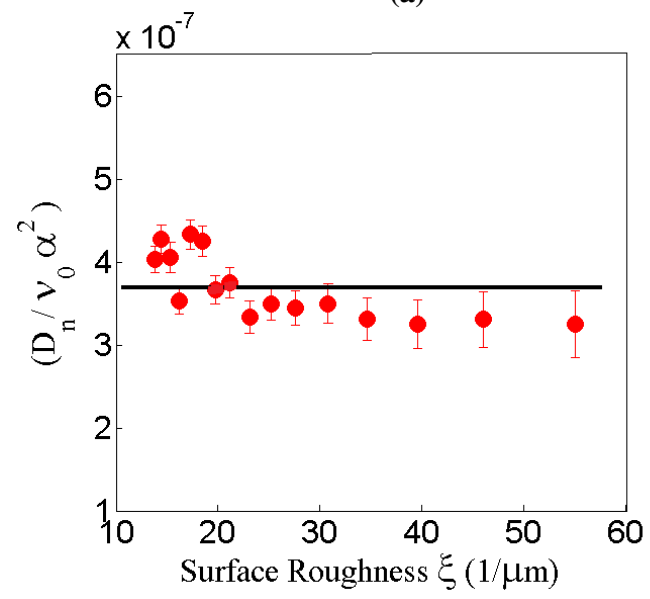

(c)

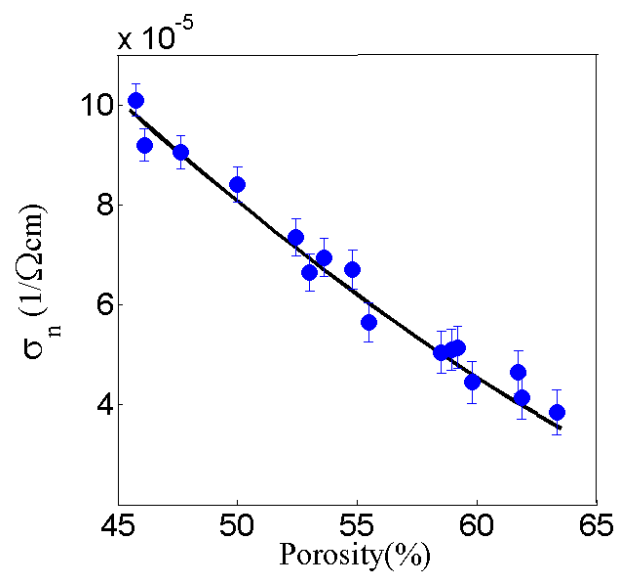

(b)

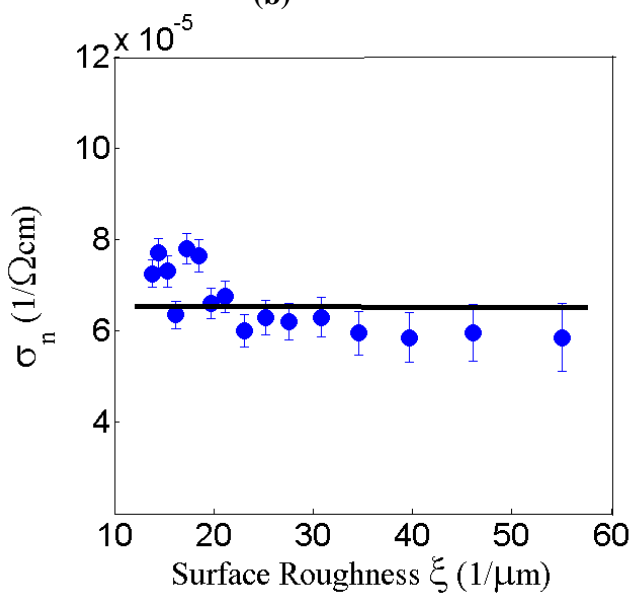

(d)

Figure 2. Chemical diffusion coefficient $(a, c)$ and conductivity (b, d) of the porous media at constant Fermi level ($0.7 \mathrm{eV}$ ) as a function of porosity and surface roughness obtained by Equation 17 (Solid lines) and the simulation (point)

Table 1. The values of constants used for the simulations

\begin{tabular}{|c|c|c|}
\hline & Value & Reference \\
\hline$n_{p h}\left(\mathrm{~cm}^{-3}\right)$ & $2.5 \times 10^{15}$ & {$[11,20]$} \\
\hline $\boldsymbol{n}_{t r}\left(n m^{-3}\right)$ & 3 & {$[11,20]$} \\
\hline$T_{0}(K)$ & 600 & {$[7,24]$} \\
\hline$T(K)$ & 300 & Room Temp. \\
\hline $\boldsymbol{v}_{\mathbf{0}}(\mathrm{THz})$ & 1.5 & {$[5]$} \\
\hline$n_{p h}^{s}\left(c m^{-2}\right)$ & $7 \times 10^{9}$ & [20] \\
\hline$E_{t r}(e V)$ & -0.2 & [20] \\
\hline$E_{f}(e V)$ & -0.7 & {$[5,24]$} \\
\hline$\alpha(n m)$ & 0.5 & {$[11,20]$} \\
\hline$n_{0}^{s}\left(n m^{-2}\right)$ & 1 & {$[5,7]$} \\
\hline$d_{r}(n m)$ & 18 & {$[3,4]$} \\
\hline
\end{tabular}

Random walk simulation based on hopping model is also carried out at a constant photo-charge density. As mentioned in the simulation implementation part, traps with a given density of $n_{\text {tr }}$ are 
distributed in the porous media. Trap filling is affected by changing the Fermi level. Consequently for the simulation at a constant Fermi level, traps with energy lower than Fermi level is filled and electron transport occurs between the unfilled traps. On the other hand, for simulations at a constant photo-charge, $\mathrm{n}_{\mathrm{ph}}$ of the traps with the minimum energies are filled.

Figure 3 demonstrates the results of the simulations for diffusion coefficient ( $3 a$ ) and conductivity (3b) as a function of surface roughness at constant photo-charge. Curves are obtained by Equation 19. As shown in this figure there are a good agreement between the results of simulation and theory. It is worth mentioning that the chemical diffusion coefficient and conductance depends on the surface roughness under constant photo-charge condition which is in agreement with the reported experimental results ${ }^{6,29}$.

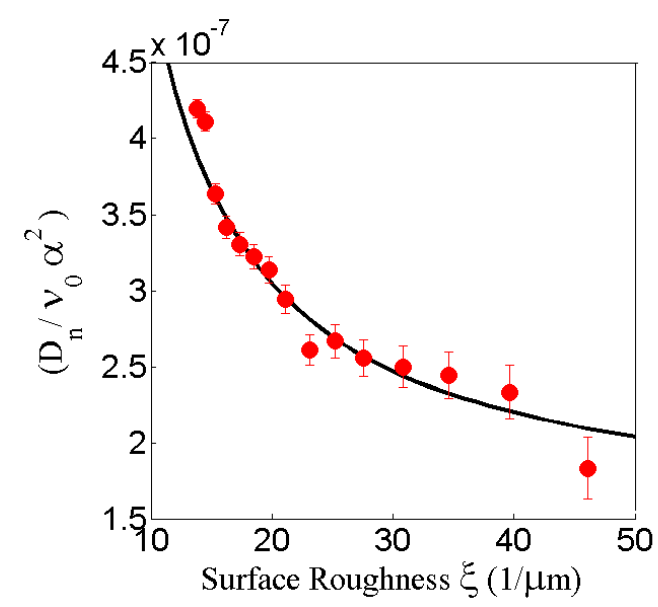

(a)

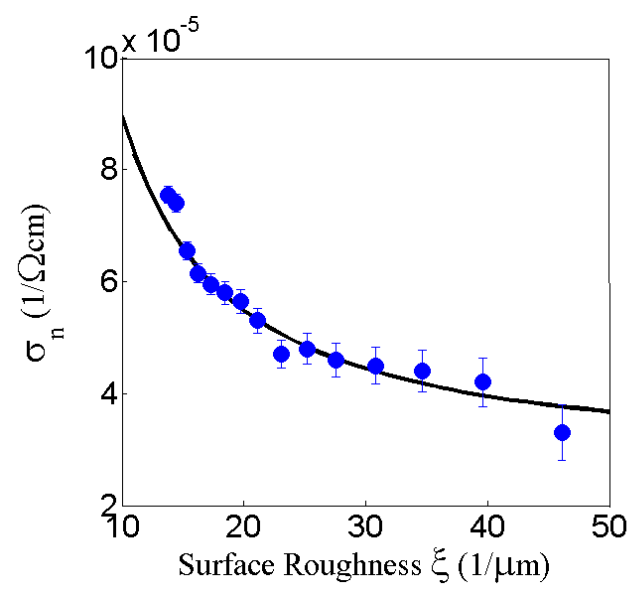

(b)

Figure 3. Chemical diffusion coefficient (a) and conductivity (b) of the porous media at constant photo-charge density versus surface roughness obtained by Equation 19 (Solid lines) and the simulation (point)

\section{Conclusion}

In conclusion, we have derived a useful working formula for the morphological dependency of the chemical diffusion coefficient and conductance of electrons in nano-structured solar cells using the hopping model. Although dependency of the chemical diffusion coefficient on the morphological parameters has been obtained previously by the random walk simulations based on multiple trapping model, we used the hopping transport model here to obtain a mathematical form for the morphological dependency of the diffusion coefficient. Equations 17 and 19 are introduced here as equations giving the chemical diffusion coefficient as a function of both morphological parameters (porosity and surface roughness) and electronic parameters (Fermi level and charge densities). We hope that this work will encourage further experimental work focused on the morphological dependency of the diffusion coefficient.

\section{Acknowledgments}

We would like to thank the Iran National Science Foundation (INSF) for partial financial support. 
Reference

[1] Bavarian, M.; Nejati, S.; Lau, K. K.; Lee, D.; Soroush, M. Theoretical and Experimental Study of a Dye-Sensitized Solar Cell. Industrial \& Engineering Chemistry Research, 2013 53(13), 52345247.

[2] Smolin, Y. Y.; Nejati, S.; Bavarian, M.; Lee, D.; Lau, K. K.; Soroush, M. Effects of Polymer Chemistry on Polymer-Electrolyte Dye Sensitized Solar Cell Performance: A Theoretical and Experimental Investigation. Journal of Power Sources, 2015 274, 156-164.

[3] Soga, T. Nanostructured Materials for Solar Energy Conversion, $1^{\text {st }}$ ed.; Elsevier Science: Amsterdam, The Netherlands, 2006.

[4] Gregg, B. A. Excitonic Solar Cells. J. Phys. Chem. B 2003, 107, 4688-4698

[5] Kopidakis, N.; Neale, N. R.; Zhu, K.; van de Lagemaat, J.; Frank, A. Spatial location of transport-limiting traps in TiO2 nanoparticle films in dye-sensitized solar cells. J. Appl. Phys. Lett. 2005, 87, 202106.

[6] Nakade, S.; Saito, Y.; Kubo, W.; Kitamura, T.; Wada, Y.; Yanagida, S. Influence of $\mathrm{TiO}_{2}$ Nanoparticle Size on Electron Diffusion and Recombination in Dye-Sensitized $\mathrm{TiO}_{2}$ Solar Cells. J. Phys. Chem. B 2003, 107, 8607-8611

[7] Anta, J. A.; Morales-Fló rez, V. Combined Effect of Energetic and Spatial Disorder on the Trap-Limited Electron Diffusion Coefficient of Metal-Oxide Nanostructures. J. Phys. Chem. C 2008, 112, 10287-10293.

[8] Abdi, N.; Abdi, Y.; Nedaaee-Oskoee, E.; Sajedi, M. Electron diffusion in trap-contained 3D porous nanostructure: simulation and experimental investigation. J. Nanopart. Res. 2014, 16, 2308.

[9] Ansari-Rad, M.; Abdi. Y.; Arzi, E. Monte Carlo Random Walk Simulation of Electron Transport in Dye-Sensitized Nanocrystalline Solar Cells: Influence of Morphology and Trap Distribution. J. Phys. Chem. C 2012, 116, 3212-321.

[10] Cass, M. J.; Qiu, F. L.; Walker, A. B.; Fisher, A. C.; Peter, L. M. Influence of Grain Morphology on Electron Transport in Dye Sensitized Nanocrystalline Solar Cells. J. Phys. Chem. B 2003, 107, 113-119.

[11]Cass, M. J.; Walker, A. B.; Martinez, D.; Peter, L. M. Grain Morphology and Trapping Effects on Electron Transport in Dye-Sensitized Nanocrystalline Solar Cells. J. Phys. Chem. B 2005, 109, 5100-5107. 
[12]Bisquert, J. Chemical Diffusion Coefficient of Electrons in Nanostructured Semiconductor Electrodes and Dye-Sensitized Solar Cells. J. Phys. Chem. B 2004, 108, 2323-2332.

[13] Tessler, N.; Preezant, Y.; Rappaport, N.; Roichman, Y. Charge Transport in Disordered Organic Materials and Its Relevance to Thin-Film Devices: A Tutorial Review. Adv. Mater. 2009, $21,2741-2761$.

[14] Jamnik. J.; Maier, Generalised equivalent circuits for mass and charge transport: chemical capacitance and its implications. J. Phys. Chem. Chem. Phys. 2001, 3, 1668.

[15] Bisquert, Chemical capacitance of nanostructured semiconductors: its origin and significance for nanocomposite solar cells. J. Phys. Chem. Chem. Phys. 2003, 5, 5360.

[16] van de Lagemaat, J.; Frank, A. J. Nonthermalized Electron Transport in Dye-Sensitized Nanocrystalline $\mathrm{TiO}_{2}$ Films: Transient Photocurrent and Random-Walk Modeling Studies. J. Phys. Chem. B 2001, 105, 11194-11205.

[17] Fabregat-Santiago, F.; Mora-Sero’, I.; Garcia-Belmonte, G.; Bisquert, J. Cyclic Voltammetry Studies of Nanoporous Semiconductors. Capacitive and Reactive Properties of Nanocrystalline $\mathrm{TiO}_{2}$ Electrodes in Aqueous Electrolyte. J. Phys. Chem. B 2003, 107, 758

[18] Bisquert, Interpretation of electron diffusion coefficient in organic and inorganic semiconductors with broad distributions of states. J. Phys. Chem. Chem. Phys. 2008, 10, 31753194

[19] van der Ven, A.; Ceder, G.; Asta, M.; Tepesch, P. D. First-principles theory of ionic diffusion with nondilute carriers. Phys. Rev. B, 2001, 64, 184307.

[20] Gomer, R. Diffusion of adsorbates on metal surfaces. Rep. Prog. Phys., 1990, 53, 917.

[21] Ala-Nissilay, T.; Ferrando, R.; Yingz, S. C. Collective and Single Particle Diffusion on Surfaces. Adv.Phys.2002, 51, 949-1078.

[22] Bisquert, J. Hopping Transport of Electrons in Dye-Sensitized Solar Cells. J. Phys. Chem. C 2007, 111, 17163.

[23] Miller, A.; Abrahams, S. Impurity Conduction at Low Concentrations. Phys. Rev. 1960, 120, 745.

[24]Gonzalez-Vazquez, J. P. Anta, J. A.; Bisquert, Random walk numerical simulation for hopping transport at finite carrier concentrations: Diffusion coefficient and transport energy concept. $J$. Phys. Chem. Chem. Phys. 2009,11, 10359-10367.

[25] Ziff R M, Flux to a trap. J. Stat. Phys. 1991, 65 1217-33. 
[26] Javadi, M.; Abdi, Y. Monte Carlo random walk simulation of electron transport in confined porous $\mathrm{TiO} 2$ as a promising candidate for photo-electrode of nano-crystalline solar cellsJ. App. Phys. 2015 118, 0643041-0643047.

[27] ] Javadi, M.; Abdi, Y.; Arzi, E. Local collection efficiency in the nano-crystalline solar cells. Solar Energy 2016 133, 549-555.

[28] Arthur J. Frank, Nikos Kopidakis, Jao van de Lagemaat; Electron in nanostructured TiO2 solar cell:transport, recombination and photovoltaic properties. Coord Chem Rev. Coord. Chem. Rev 2004, 248, 1165-1179

[29] Kopidakis, N.; Neale, N. R.; Zhu, K.; van de Lagemaat, J.; Frank, A. Spatial location of transport-limiting traps in $\mathrm{TiO} 2$ nanoparticle films in dye-sensitized solar cells. J. Appl. Phys. Lett. 2005, 87, 202106. 